\title{
Automedicação induzida pelos fatores midiáticos: uma abordagem no ambiente acadêmico
}

\author{
Self-medication induced by media factors: an approach in the academic environment
}

La automedicación inducida por factores mediáticos: un enfoque en el entorno académico

Tatiana Naiana Rodrigues dos Santos Porto', Magda Denise da Silva Barbosa1, Marcileide Lima do Carmo ${ }^{1}$, Benedito Pereira de Sousa Neto ${ }^{1}$, Nilton Andrade Magalhães ${ }^{1}$, Luciana Stanford Baldoino ${ }^{*}$,

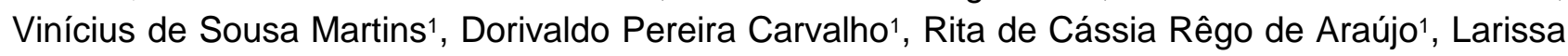
Stanford Baldoino Banks².

\section{RESUMO}

Objetivo: Avaliar a automedicação influenciada pela mídia e fatores envolvidos entre estudantes de enfermagem. Métodos: Trata-se de um estudo quantitativo, descritivo e exploratório com 49 acadêmicos do curso de enfermagem. A organização dos dados deu-se por meio de planilhas do programa Microsoft Excel ${ }^{\circledR}$ apresentados em tabelas e foram analisados sob a forma de estatística descritiva, aplicando-se o cálculo de porcentagem simples. Resultados: Dos entrevistados, $90 \%$ era do sexo feminino, com faixa etária predominante entre 18 e 24 anos. Sendo que o maior número de acadêmicos participantes solteiro (49\%). (84\%) afirmaram ter feito automedicação no último trimestre, justificando ter sido por dificuldades de acesso aos serviços de saúde pública (48\%). Os medicamentos mais usados são os anti-inflamatórios e analgésicos. Os efeitos colaterais mais observados foram cefaleia e náuseas, apesar de (77,5\%), afirmarem não ter apresentado nenhuma complicação medicamentosa. Em relação às propagandas com medicamentos (59\%) afirmaram ser um fator determinante da automedicação. Dos pesquisados $(37 \%)$ usaram medicamento para potencializar o seu desempenho acadêmico e (28\%) utilizou automedicação para fins estéticos. Conclusão: Os fatores midiáticos são considerados recursos que aumentam a procura por medicamentos em busca de alívio rápido e muitas vezes paliativo de sintomas que tendem a surgir esporadicamente.

Palavras-chave: Automedicação, Mídia, Desempenho acadêmico, Autocuidado, Enfermagem.

\begin{abstract}
Objective: Analyzed the media influence for self-medication among nursing students. Methods: A study quantitative, descriptive and exploratory research with 49 nursing students. The organizations of data collection were through Microsoft Excel ${ }^{\circledR} 2010$ this presented in tables and analyzed in the form of descriptive statistics, applying the simple percentage calculation. Results: Of 49 students were interviewed, of whom $90 \%$ were female, with a predominant age group between 18 and 24 years old. The largest numbers of participating students are single (49\%). (84\%) of the students said that had self-medication in the last trimester, justifying to have been due to difficulties in accessing public health services (48\%). The most commonly used drugs are anti-inflammatory and analgesics. The most observed collateral effects were headache and nausea, although (77.5\%) reported no drug complications. Regarding drug advertisements, $(59 \%)$ said to be a determinant of self-medication. Of respondents (37\%) used medication to boost their academic performance and (28\%) used self-medication for aesthetic purposes. Conclusion: Mediatic factors are considered to be resources that increase the demand for drugs in search of quick and often palliative relief of symptoms that tend to arise sporadically.
\end{abstract}

Keywords: Self-medication, Media, Academic performance, Self-care, Nursing.

\footnotetext{
${ }^{1}$ Faculdade de Ensino Superior Múltiplo (IESM), Timon-MA. *E-mail: Isbaldoino@hotmail.com

2 Faculdade R. Sá (IESRSA), Picos-PI.
}

SUBMETIDO EM: 12/2019

ACEITO EM: 1/2020

PUBLICADO EM: $3 / 2020$ 


\section{RESUMEN}

Objetivo: Analizó la influencia de los medios para la automedicación entre los estudiantes de enfermería. Métodos: Estudio de investigación cuantitativa, descriptiva y exploratoria con 49 estudiantes de enfermería. Las organizaciones de recolección de datos fueron a través de Microsoft Excel® 2010, esto se presentó en tablas y se analizó en forma de estadísticas descriptivas, aplicando el cálculo porcentual simple. Resultados: De 49 estudiantes entrevistados, de los cuales el $90 \%$ eran mujeres, con un grupo de edad predominante entre 18 y 24 años. El mayor número de estudiantes participantes son solteros (49\%). (84\%) de los estudiantes dijeron que se automedicaron en el último trimestre, lo que justificó que se debieron a dificultades para acceder a los servicios de salud pública (48\%). Los fármacos más utilizados son los antiinflamatorios y analgésicos. Los efectos colaterales más observados fueron dolor de cabeza y náuseas, aunque (77.5\%) no informaron complicaciones farmacológicas. Con respecto a los anuncios de drogas, (59\%) se dice que es un determinante de la automedicación. De los encuestados (37\%) usaron medicamentos para mejorar su rendimiento académico y $(28 \%)$ usaron automedicación con fines estéticos. Conclusion: Los factores mediáticos se consideran recursos que aumentan la demanda de medicamentos en busca de un alivio rápido y a menudo paliativo de los síntomas que tienden a surgir esporádicamente.

Palabras-clave: Automedicación, Medios audiovisuales, Rendimiento académico, Autocuidado, Enfermería.

\section{INTRODUÇÃO}

A automedicação consiste no uso de medicamento não prescrito por profissionais habilitados para tratar distúrbios patológicos ou sintomas auto-diagnosticados. É um fenômeno complexo, que pode estar associado com vários fatores, entre eles a angústia pela cura da doença, a dificuldade de acesso da população aos serviços de saúde e a ausência de informação a respeito dos riscos relacionados a essa prática, à facilidade de acesso ao medicamento e a ausência de controle por parte dos órgãos de fiscalização (HOFFMANN AMM, et al., 2017).

Vale enfocar que o Brasil é um dos maiores consumidores de medicamento do mundo, com o mercado que alcança aproximadamente 22,1 bilhões de dólares anualmente. Dados da Federação Brasileira das Redes Associativistas de Farmácias, mostram que o Brasil possui aproximadamente 65 mil farmácias e drogarias, uma proporção de 3,3 farmácias para cada 10 mil habitantes. No entanto, a Organização Mundial da Saúde (OMS) preconizou em 2009 que a proporção de farmácia seja de uma para cada 10.000 habitantes, no Brasil, esse número é três vezes maior (LEITE ICP, et al., 2016).

De acordo com Hoffmann AMM et al. (2017), os principais fatores que colaboram para a permanência da automedicação, apesar da legislação criada há quase vinte anos, seria a influência da propaganda, uso de prescrições antigas, orientação de funcionários de farmácias, amigos, vizinhos e familiares, armazenamento de medicamentos em casa, além da cultura do autocuidado com a saúde.

Este número de farmácias aumentam o consumo descontrolado de medicamentos devido ao fácil acesso para a compra, vale destacar que, mais de $50 \%$ das medicações são vendidas de forma ilegal e sem prescrição médica. Aproximadamente, $76,4 \%$ da população brasileira fazem ou já fizeram em algum momento da vida uso de medicamentos indicados por familiares, amigos ou vizinhos e cerca de $32 \%$ aumentaram a dose do medicamento por conta própria (DOMINGUES PH, et al., 2015).

Considera-se os acadêmicos de enfermagem representam um grupo significante para este tipo de pesquisa, corroborando com as informações mencionadas, já que estes necessitam adotar já durante o período de estágio uma postura física e psicológica rigorosa frente a dor de seus pacientes, pois a profissão almejada desenvolve capacidades e habilidades ligadas diretamente a essa problemática (SOUZA DRP e NETA ME, 2016).

Além disso, a automedicação é considerada um problema de saúde pública mundial, sendo que alguns fatores contribuem para a sua difusão, dentre estes fatores tem-se a questão cultural. No Brasil, o uso de plantas medicinais por nativos favoreceu culturalmente a automedicação. Sabe-se que o hábito da utilização 
de medicamento por conta próprio pode ter originado a partir desse recurso terapêutico e isso tornou-se uma prática comum vivenciada por várias gerações ao longo do tempo (DUTRA RC, et al., 2016).

Tendo em vista que a automedicação no Brasil representa $35 \%$ do consumo de fármacos, sendo que $27 \%$ das intoxicações medicamentosas e $16 \%$ dos óbitos são resultantes dessa prática. Os hospitais gastam em média de 15 a $20 \%$ de seus orçamentos para lidar com as complicações causadas pelo uso indevido dos mesmos (VIEIRA DM e CAVEIÃO C, 2016). É pertinente enfocar que a automedicação é praticada desde os primórdios da civilização, em que os povos usavam ervas de modo empírico para cura de alguma enfermidade (ANGELO T, et al., 2014).

Esta prática pode ser classificada basicamente de três formas, sendo a primeira diretamente relacionada a questão cultural, quando se faz uso de medicamento a partir do conhecimento adquirido ao longo do tempo, e que é passado através de gerações. A segunda condição seria a orientada, quando o paciente já possui conhecimentos prévios sobre os medicamentos que pretende utilizar, e a terceira é a induzida, quando o uso de medicamentos é realizado devido à influência de campanhas publicitárias com fins comerciais (IURAS A, et al., 2016).

Vários fatores contribuem para prática da automedicação, um que merece destaque é a dificuldade de acesso aos serviços de saúde especializados, outro fator determinante é a falta de fiscalização por parte dos órgãos públicos isso possibilita a venda indiscriminada de medicamento somado a esses outros fatores a facilidade de acesso aos medicamentos em estabelecimentos farmacêuticos.

A mídia também influência bastante o uso indiscriminado de medicamento, algumas vezes, a propaganda com os fármacos, que geralmente enfatizam os benefícios e minimizam as possíveis reações adversas e outros riscos, acaba sendo a única informação de referencial que o paciente tem acesso (OLIVEIRA HJP, et al., 2017).

Nesse sentido, justifica-se a relevância deste estudo por entendermos que o fenômeno da automedicação é um típico problema verificado ao longo das gerações e é um indicador indireto da qualidade dos serviços de saúde. A propaganda em massa e a facilidade de acesso a medicamentos são estímulos ao uso indiscriminado e, por conseguinte, um risco em potencial para o comprometimento da saúde física e mental. Devido à herança cultural, a prática é considerada inofensiva. A busca pelo alívio imediato de sintomas e o acesso fácil aos medicamentos contribuem para elevação do índice de dependência medicamentosa.

Em face do exposto, o estudo promove a incorporação de medidas educativas, quanto ao uso irracional de medicamentos. Uma das formas de tentar reverter esse quadro, que é preocupante, foi investigando o perfil epidemiológico, as causas atribuídas às práticas do autodiagnóstico e da automedicação. Diante do exposto, o presente trabalho teve como objetivo discutir a problemática do uso indiscriminado de medicamentos por estudantes de enfermagem influenciado pela mídia e fatores envolvidos.

\section{MÉTODOS}

Trata-se de um estudo quantitativo, exploratório e descritivo. A pesquisa foi realizada por meio de questionário semiestruturado com perguntas fechadas e de múltipla escolha, aplicado aos acadêmicos do curso de enfermagem. Foram incluídos na pesquisa, estudantes de enfermagem a partir do segundo ano de faculdade que corresponde a $40 \%$ da carga horária da graduação. A justificativa da escolha deste público foi o fato de que a partir da etapa supracitada, as atividades são ambientadas não apenas nos limites territoriais acadêmicos, mas também na área hospitalar, exigindo habilidades cognitivas mais abrangentes, já que eles estarão expostos à realidade social profissional, sobrecarregando as funções física e mental.

Os dados foram coletados e organizados em planilhas do software Microsoft Excel® 2010 apresentados em tabelas e analisados sob a forma de estatística descritiva, aplicando o cálculo de porcentagem simples. Já as variáveis do estudo foram relacionadas com aspectos sociais e econômicos direcionadas aos acadêmicos de enfermagem: gênero, idade, estado civil, renda familiar. Vale destacar que as variáveis relacionadas sobre o uso indiscriminado de medicamentos foram: uso de medicamentos no último trimestre, reações adversas, uso de medicamentos com prescrição médica. 
Tendo em vista, os citados preceitos, todos os participantes assinaram o termo de consentimento livre e esclarecido, onde tiveram a garantia do sigilo dos seus nomes e a liberdade de recusar ou retirar seu consentimento em qualquer fase da pesquisa.

A abordagem utilizada durante a aplicação do questionário respeitou os preceitos estabelecidos no Conselho Nacional de Saúde, resolução no 466/2012, já que está pesquisa envolve o contato direto com seres humanos. Pelo fato de a escolha do local de pesquisa ser em uma instituição de ensino, priorizamos a realização do estudo no horário de intervalo, visto que este facilitou a aquiescência dos indivíduos e evitou interrupções das atividades curriculares. O projeto de pesquisa foi submetido à Plataforma Brasil e sua aplicação se deu mediante autorização sob o parecer do Comitê de Ética de Pesquisa (CEP) do Hospital Getúlio Vargas tendo CAAE n 79613717.3.0000.5613.

\section{RESULTADOS}

A pesquisa teve participação consentida de 49 estudantes regularmente matriculados, sendo que $90 \%$ (44) dos participantes foram do sexo feminino, a faixa etária predominante foi de 18 a 24 anos representando 47\%. (Tabela 1).

Tabela 1 - Distribuição dos estudantes de enfermagem de acordo com o sexo e a faixa etária. Timon (MA), 2019.

\begin{tabular}{ccc}
\hline Sexo & $\mathbf{N}$ & $\%$ \\
\hline Feminino & 44 & 90 \\
Masculino & 5 & 10 \\
\hline Faixa Etária & $\mathbf{N}$ & $\%$ \\
\hline $18-24$ & 22 & 45 \\
$25-30$ & 13 & 26 \\
$31-45$ & 14 & 29 \\
\hline Total & $\mathbf{4 9}$ & $\mathbf{1 0 0}$ \\
\hline
\end{tabular}

Fonte: Porto TNRS, et al., 2019.

Em relação ao estado civil, 24 acadêmicos declaram ser solteiros o equivalente a $49 \%$ dos entrevistados. Seguidos dos que disseram ser casados, 21 acadêmicos representados $43 \%$. A renda familiar predominante foi de dois salários mínimos de referência, equivalendo $35 \%$ dos pesquisados. Os que possuem renda familiar de um e três salários mínimos representam cada grupo $26,5 \%$ (Tabela 2).

Tabela 2 - Distribuição dos estudantes de enfermagem de acordo com o estado civil e renda salarial. Timon (MA), 2019.

\begin{tabular}{ccc}
\hline Estado civil & $\mathbf{N}$ & $\%$ \\
\hline Solteiro & 24 & 49 \\
Casado & 21 & 43 \\
União estável & 4 & 8 \\
\hline Renda salarial & $\mathbf{N}$ & $\%$ \\
\hline Um salário minímo & 13 & 26,5 \\
Dois salários & 17 & 35 \\
Três salários & 13 & 26,5 \\
>Três salários & 6 & 12 \\
\hline Total & $\mathbf{4 9}$ & $\mathbf{1 0 0}$ \\
\hline
\end{tabular}

Fonte: Porto TNRS, et al., 2019.

Em relação ao uso de medicamentos sem prescrição médica no último trimestre, $84 \%$ dos entrevistados afirmaram ter consumido. Somente $14 \%$ afirmaram não ter utilizado medicamentos sem orientação médica. Quando questionados sobre os motivos do uso de medicamentos sem prescrição médica, $64 \%$ responderam 
que o estresse acadêmico é o principal fator motivacional, e $36 \%$ responderam que o conhecimento acerca dos princípios farmacológicos, os outros fatores que os motivaram a se automedicarem foi a demora no atendimento no serviço público de saúde (48\%). Sendo que $21 \%$ disseram ter recebido indicação de familiares e $12 \%$ dos participantes afirmaram que a prática é principalmente impelida por questões econômicas (Gráfico 1).

Gráfico 1 - Distribuição das principais motivações que influenciam na prática da automedicaçao em estudantes de enfermagem. Timon (MA), 2019.



Fonte: Porto TNRS, et al., 2019.

Os estudantes foram questionados sobre os possíveis tipos de efeitos colaterais que os medicamentos possam ter Ihe ocasionados. Dos entrevistados, $52 \%$ responderam ter apresentado sonolência, $16 \%$ cefaleia, $8 \%$ relataram que nenhum sintoma foi notado. No entanto, $12 \%$ já apresentaram náuseas e os demais (12\%) afirmaram que já apresentaram mais de um sintoma (sonolência, náuseas e desconforto abdominal) proveniente do consumo de medicamentos sem prescrição (Tabela 3).

Tabela 3 - Distribuição do consumo de medicamentos sem prescrição médica no último trimestre e aparecimento de reações adversas e/ou piora dos sintomas. Timon (MA), 2019.

\begin{tabular}{ccc}
\hline Consumo de medicamentos no último trimestre & $\mathbf{N}$ & $\%$ \\
\hline Sim & 41 & 84 \\
Não & 7 & 14 \\
\hline Apresentou algum efeito colateral & $\mathbf{N}$ & $\%$ \\
\hline Sim & 11 & 22,5 \\
Não & 38 & 77,5 \\
\hline Total & $\mathbf{4 9}$ & $\mathbf{1 0 0}$ \\
\hline
\end{tabular}

Fonte: Porto TNRS, et al., 2019.

Quanto às complicações de saúde observadas logo após ingerir algum medicamento por conta própria, $77,5 \%$ responderam que não ter apresentado nenhuma complicação e $25 \%$ afirmaram que já apresentaram. Com relação às propagandas de medicamentos veiculadas na mídia, 59\% afirmaram que estas induzem ao aumento da prática de automedicação, $20 \%$ acreditam que deveria haver uma fiscalização mais abrangente, $9 \%$ classificam as mesmas como desnecessárias e $12 \%$ afirmaram que deveriam ser proibidas pelo Ministério da Saúde. 
No que diz respeito às classes de medicamentos mais adquiridas, observou-se que $17 \%$ dos entrevistados consomem diferentes tipos de medicações por conta própria, $40 \%$ afirmaram que sempre utilizam analgésicos, $17 \%$ consomem anti-inflamatórios com frequência e $12 \%$ utilizam antibióticos. Os acadêmicos foram entrevistados quanto ao uso de medicamentos para fins estéticos, $72 \%$ respondendo que nunca ingeriram medicações para esse fim, e $12 \%$ já usaram como catárticos e $8 \%$ termogênicos e os demais (8\%) combinaram dois ou mais fármacos.

No item que retrata o consumo de medicamentos após a realização das atividades acadêmicas, $61 \%$ responderam não ter feito uso de fármacos. É sabido que muitos acadêmicos fazem uso de medicamentos objetivando otimizar seu desempenho estudantil. Porém, o estudo mostrou que $63 \%$ (31) dos participantes nunca fizeram uso de medicamentos para potencializar o seu desempenho nas atividades acadêmicas. E que $37 \%$ (18) fizeram uso de medicamentos com essa finalidade. (Tabela 4).

Tabela 4 - Distribuição dos estudantes que fizeram combinação de medicamentos sem prescrição médica para sintomatologias diferentes. Timon (MA), 2019.

\begin{tabular}{ccc}
\hline Combinou medicamentos para sintomatologias diferentes? & $\mathbf{N}$ & $\%$ \\
\hline Sim & 19 & 39 \\
Não & 30 & 61 \\
\hline Automedicou-se após realizar alguma atividade acadêmica? & $\mathbf{N}$ & $\%$ \\
\hline Sim & 19 & 39 \\
Não & 30 & 61 \\
\hline Total & $\mathbf{4 9}$ & $\mathbf{1 0 0}$ \\
\hline
\end{tabular}

Fonte: Porto TNRS, et al., 2019.

Ao serem questionados se já haviam combinado um ou mais medicamentos para sintomatologias diferentes, $61 \%$ responderam nunca fizeram combinação medicamentosa e $39 \%$ afirmaram que sim.

Dos acadêmicos pesquisados $86 \%$ afirmaram que nunca foram diagnosticados com uma doença mais grave após se automedicar, enquanto $14 \%$ disseram que isso já aconteceu depois de praticar esse método (Tabela 5).

Tabela 5 - Distribuição dos estudantes que utilizaram medicamentos sem prescrição médica e posteriormente foram diagnosticados com doenças mais graves. Timon (MA), 2019.

\begin{tabular}{ccc}
\hline $\begin{array}{c}\text { Ingeriu medicamento achando se tratar de sintoma passageiro e foi diagnosticado } \\
\text { algo mais grave? }\end{array}$ & $\mathbf{N}$ \\
\hline Sim & 7 & 14 \\
Não & 42 & 86 \\
\hline Total & $\mathbf{4 9}$ & $\mathbf{1 0 0}$ \\
\hline
\end{tabular}

Fonte: Porto TNRS, et al., 2019.

\section{DISCUSSÃO}

O estudo descreve a prática da automedicação entre estudantes de enfermagem. A prevalência da referida prática observada (82\%) foi superior ao de outros estudos que exploram a mesma abordagem, tais como Alves DRF et al. (2019), no qual 50,78\% dos participantes afirmaram se automedicar e Gama ASM, Secoli SR (2017) que apresentou prevalência de $76 \%$.

Uma possível justificativa para essa diferença entre os estudos pode ser o tamanho da amostra nesta pesquisa ter sido inferior à amostra destes autores. Nos estudos houve a participação consentida de $200 \mathrm{e}$ 116 acadêmicos respectivamente. Enquanto esse estudo teve aquiescência de 49 acadêmicos, o que evidência os contrastes nos resultados encontrados, além disso, o recorte temporal também pode ter influenciado na diferença dos resultados. 
Trabalho publicado em 2016 mostra que na América Latina, o Brasil assume a primeira posição dentre os países que mais praticam a automedicação, ocupando a quinta posição na listagem mundial, além disso são vendidos nacionalmente de maneira indiscriminada cerca de 300 mil medicamentos por ano. Ainda de acordo com o mesmo autor, isso se explica pelo fato das drogarias serem consideradas um simples ponto de venda de medicamento, e não um estabelecimento de saúde (ARRAIS PSD, et al., 2016).

As classes de medicamentos mais referidas foram analgésicas (40\%) e anti-inflamatórios (17\%) corroborando com a pesquisa realizada por Rodrigues WP, et al. (2019) na qual analisou os fármacos mais prevalentes em estoques domiciliares, isso se justifica por serem medicamentos comercializados indiscriminadamente o que facilita a aquisição, como demonstraram.

Nesse seguimento, outro fator que pode estar diretamente interligado ao consumo expressivo de analgésicos e anti-inflamatórios entre graduandos são as queixas relativas à dor, tendo como motivação a rotina diária associada ao desgaste físico e psíquico evidenciado pelo estresse, o que causa danos à saúde observados em longo prazo (VIEIRA GCG, et al., 2016).

Por ser um fármaco com aplicabilidade em diferentes reações patológicas, o analgésico é utilizado com frequência não apenas no ambiente acadêmico, mas na população em geral, estímulo semelhante à preferência por anti-inflamatórios, que apresentam diferentes funções, com ação eficaz percebida em múltiplas sintomatologias (GAMA ASM e SECOLI SR, 2017).

O resultado encontrado tem relação direta com outra variável analisada, o gênero, pois é sabido que as mulheres são adeptas das práticas de autocuidado e apresentam-se como público prioritário das ações de saúde dos órgãos competentes que desenvolvem campanhas preventivas. Além de serem mais acometidas por cefaleias, mialgias e demais condições de dor crônica.

Em termos fisiológicos, utilizam desde o início da adolescência estes fármacos visto que minimizam o mecanismo da dor em condições inerentes à natureza feminina, tais como dismenorreia (ATHANASOPOULOS C e PITYCHOUTIS PM, 2013).

Tendo em vista, que os fatores propulsores da automedicação, segundo a opinião dos acadêmicos é a demora no atendimento no serviço público de saúde (48\%) e indicação de algum membro da família (21\%). Essas variáveis têm uma ligação direta ao quantitativo salarial, no qual predominou a alternativa referente a dois salários mínimos (35\%), seguido de um e três salários mínimos (26,5\%). Sendo que os acadêmicos cuja renda familiar é superior a três salários mínimos de referência a prática da automedicação apresentou-se menor, que pode estar relacionado ao acesso aos serviços de saúde privado (SADO E, et al., 2017).

Embora haja direitos previstos em legislação referente à saúde, é notório que a operacionalização dos serviços dessa área não atinge níveis de qualidade como atendimento condizente com os padrões de necessidade da população brasileira. Grande parcela da população possui baixo poder aquisitivo, impossibilitando-os de procurar apoio médico em instituições terapêuticas privadas e consequentemente aderirem a um plano de saúde.

O conhecimento empírico também foi classificado como uma motivação plausível para a disseminação da prática da automedicação segundo a ótica dos acadêmicos entrevistados, já que a opinião de pessoas que possuem parentesco com os mesmos, influencia na aquisição de fármacos (GAMA ASM e SECOLI SR, 2017).

No trabalho publicado por luras A et al. (2016) mostra ainda que a maioria dos universitários entrevistados $88 \%$ afirmaram não possuir conhecimento suficiente para se automedicar. Por outro lado, o autor observou que $74 \%$ dos entrevistados alegaram possuir conhecimento sobre as reações adversas e efeitos colaterais causados pelos medicamentos.

Segundo o autor do trabalho este resultado foi surpreendente porque o estudo foi realizado com acadêmicos da área de saúde. No entanto, a recomendação de leigos pode causar transtornos à saúde, pois pode haver uma interpretação errônea dos critérios de posologia e dosagem o que não aconteceria por um profissional especializado, muitos que escolhem esta prática recebem orientações e indicações de familiares, colegas e pessoas do círculo social que já fizeram uso de medicamentos sem a recomendação de um profissional, o que pode resultar em problemas graves e até mesmo fatais (GUALANO MR, et al., 2014). 
Jesus AP e Yoshida NC et al. (2013) descreveram em seu trabalho que quando adentram a faculdade, os estudantes precisam se adequar às atividades curriculares e extracurriculares que por vezes exigem uma capacidade cognitiva acentuada que requer maior concentração e determinação de prioridades, sendo assim, é necessário estabelecer tempo para efetuá-las, impossibilitando os mesmos de procurarem orientação médica para tratar determinadas sintomatologias que venham a apresentar, o que torna a automedicação uma prática mais rápida e econômica.

Em contraste com a pesquisa de Pessanha FF et al. (2014), que avaliou a prevalência do uso de metilfenidato por universitários de Campos de Goytacazes, verificou que $60 \%$ dos estudantes relataram uso esporádico de metilfenidato, e que $92 \%$ utilizaram essa substância para potencializar o estudo para provas. A parcela dos entrevistados formada por indivíduos casados correspondeu a $43 \%$ o que justifica o desgaste associado às responsabilidades exigidas por esses acadêmicos, como por exemplos os cuidados com o lar.

Apesar das respostas encontradas no estudo terem sido em sua maioria, negativas, a combinação de princípios ativos na sociedade é bastante comum, e isso pode provocar um efeito denominado de interação medicamentosa, visto que o consumo de fármacos com ações distintas pode contribuir para o aumento de reações adversas, vindo a reduzir a eficiência da terapêutica aplicada (ALVES DRF, et al., 2019).

No que tange às propagandas que envolvem medicamentos, $59 \%$ dos acadêmicos consideram essas como estímulos ao aumento da prática de automedicação. Essa resposta tem relação direta com a menor faixa etária dos pesquisados, pois $47 \%$ dos entrevistados tinham idades entre 18 e 24 anos, acadêmicos pertencentes à geração que tem relação mais próxima e constante com os recursos virtuais tecnológicos, principalmente as mídias sociais, além disso, a rotina diária da maioria dos jovens encontra-se sobreposta aos aparatos digitais no cenário atual (CARSONI LMM e JUNIOR DA, 2018).

Os membros dessa geração terão acesso a um índice maior de conteúdos e ferramentas ligadas aos mais variados temas, inclusive sobre medicamentos, pois a indústria farmacêutica disponibiliza acesso facilitado de compra e venda de produtos terapêuticos, terceirizando informações importantes ligadas à saúde, o que torna esse público mais vulnerável às consequências advindas dessa prática (SELVARAJ K, et al., 2014).

Essa opinião pode ser explicada pelo fato do Brasil ser um país emergente em que a automedicação é vista de forma mais preocupante, onde as condições de saúde são limitadas se comparadas às nações desenvolvidas, o que motiva a população a procurar meios inseguros para manter e recuperar a saúde (SELVARAJ K, et al., 2014).

\section{CONCLUSÃO}

Os fatores midiáticos são considerados recursos que aumentam a procura por medicamentos em busca de alívio rápido e muitas vezes paliativo de sintomas que tendem a surgir esporadicamente. Nesta pesquisa, (84\%) dos estudantes afirmaram terem se automedicado no último trimestre,tendo a adificuldade de acesso aos serviços de saúde como justificativa, já em relação aos medicamentos mais utilizados, os analgésicos e os anti-inflamatórios foram os mais citados, os efeitos colaterais mais citados foram a cefáleia e náuseas e as propagandas de medicamentos foi u fator determinante na escolha desta prática. Espera- se que este estudo subsidie estratégias de conscientização da prática acerca dos danos da automedicação, bem como incentive futuras pesquisas sobre o tema.

\section{REFERÊNCIAS}

1. ATHANASOPOULOS C, PITYCHOUTIS PM. Is drug utilization in Greece sex dependent? A population-based sudy. Basic Clin. Pharmacol.Toxicol. 2013; 112 (Suppl): 55-62.

2. ANGELO T, et al. Utilização de plantas medicinais e fitoterápicas por idosos. Revista Eletrônica da Fainor. 2014; 7(1): 18-31.

3. ALVES DRF, et al. Self-medication: practice among nursing undergraduates. Journal Nursing UFPE online. 2019; 13(2): 363-70.

4. ARRAIS PSD, et al. Prevalência da automedicação no Brasil e fatores associados. Rev Saúde Pública. $2016 ; 50$ (Supl 2): $1-13$. 
5. CARSONI LMM, JUNIOR DA. Marketing farmacêutico: relação das publicidades televisivas com a automedicação. Visão Acadêmica, 2018; 19(4): 55-66.

6. DOMINGUES PH, et al. Prevalência da automedicação na população adulta do Brasil: revisão sistemática. Rev. Saúde Pública. 2015; 3(2):1-8.

7. DUTRA RC, et al. Pharmacological studies, drug discovery, challenges and perspectives, Journal de Medicinal plants in Brazil. 2016; 112 (Suppl): 12-15.

8. GUALANO MR, et al. Use of self-medication among adolescents: a systematic review and meta-analysis. European journal of public health. 2014; 7(1): 1-7.

9. GAMA ASM, SECOLI SR. Automedicação em estudantes de enfermagem do Estado do Amazonas - Brasil. Revista Gaúcha de Enfermagem. 2017; 38(1): e65111.

10. HOFFMANN AMM, et al. Automedicação entre acadêmicos de Enfermagem. Revista Eletrônica Acervo Saúde. 2017; Suppl (9): 842-848.

11. IURAS A, et al. Prevalência da automedicação entre estudantes da Universidade do Estado do Amazonas (Brasil), Revista Portuguesa de Estomatologia, Medicina Dentária e Cirurgia Maxilofacial. 2016; 57 (Suppl): 104-111.

12. JESUS AP, YOSHIDA NC. Prevalência da automedicação entre acadêmicos de farmácia, medicina, enfermagem e odontologia. Revista Estudos, 2013; 40(2): 123-131.

13. LEITE ICP, et al. Automedicação em acadêmicos: um estudo transversal. Boletim Informativo Geum. 2016; 7(1): 1927.

14. OLIVEIRA HJP, et al. Educação em saúde como forma preventiva do uso indiscriminado dos antibióticos. Revista Saúde, 2017; 11(1): 52- 58.

15. PESSANHA FF, et al. Prevalência do uso de metilfenidato por universitários de Campos de Goytacazes. Revista Vértices, 2014; 16(1): 77-86.

16. RODRIGUES WP, et al. La automedicación entre los profesionales de la salud en Fátima/Bahia. Revista Eletrônica Acervo Saúde. 2019; 11(12): e534.

17. SADO E, et al. Epidemiology of self-medication with modern medicines among health care professionals in Nekemte town, western Ethiopia. BMC Res Notes, Nekemte. 2017; 10(4): 533.

18. SELVARAJ K, et al. Prevalence of self-medication practices and its associated factors in Urban Puducherry, India: Perspectives in Clinical Research, 2014; 5(1): 32-37.

19. SOUZA DRP, NETA ME. Automedicação por profissionais e acadêmicos da área da saúde: uma revisão de literatura. Revista da Universidade Vale do Rio Verde, 2016; 14(2): 965-974.

20. VIEIRA DM, CAVEIÃO C. Perfil das intoxicações medicamentosas no estado de São Paulo na perspectiva da vigilância sanitária. Revista Saúde e Desenvolvimento, 2016; 23(5): 32-53.

21. VIEIRA GCG, et al. Uso de psicotropicos pelo enfermeiro: sua relação com o trabalho. Cinergis, 2016; 17(3): 191195. 